\title{
Railway Wheel Flat Detection Based on Improved Empirical Mode Decomposition
}

\author{
Yifan Li, ${ }^{1}$ Jianxin Liu, ${ }^{2}$ and Yan Wang ${ }^{1}$ \\ ${ }^{1}$ Department of Mechanical Engineering, Southwest Jiaotong University, Chengdu 610031, China \\ ${ }^{2}$ Traction Power State Key Laboratory, Southwest Jiaotong University, Chengdu 610031, China \\ Correspondence should be addressed to Yifan Li; liyifan@home.swjtu.edu.cn
}

Received 6 November 2015; Revised 26 February 2016; Accepted 15 March 2016

Academic Editor: Sakdirat Kaewunruen

Copyright (c) 2016 Yifan Li et al. This is an open access article distributed under the Creative Commons Attribution License, which permits unrestricted use, distribution, and reproduction in any medium, provided the original work is properly cited.

\begin{abstract}
This study explores the capacity of the improved empirical mode decomposition (EMD) in railway wheel flat detection. Aiming at the mode mixing problem of EMD, an EMD energy conservation theory and an intrinsic mode function (IMF) superposition theory are presented and derived, respectively. Based on the above two theories, an improved EMD method is further proposed. The advantage of the improved EMD is evaluated by a simulated vibration signal. Then this method is applied to study the axle box vibration response caused by wheel flats, considering the influence of both track irregularity and vehicle running speed on diagnosis results. Finally, the effectiveness of the proposed method is verified by a test rig experiment. Research results demonstrate that the improved EMD can inhibit mode mixing phenomenon and extract the wheel fault characteristic effectively.
\end{abstract}

\section{Introduction}

Wheel flat is the most common local surface defect in railway wheels. It can result in cyclic wheel-rail impact during the running process and cause coupled vibration in the entire vehicle-track system [1]. Wheels with this fault affect the running safety significantly and cause further damage to the wheels and rails. Consequently, it is of great interest among researchers to find out effective methods for early detection and identification of wheel flats.

Large volume of methods has been developed over the decades for this purpose. There is a common way to detect wheel flats, which is the measurement of wheel-rail impact forces in an instrumented rail [2-4]. Acceleration sensors are also fixed on the rail instead of strain gauges or fiber Bragg grating to measure rail vibration $[5,6]$. Apart from the above methods, some optical, mechanical, and supersonic systems are also attempted to apply in the identification of wheel flat.

The aforementioned monitoring methods are all arranging transducers on or nearby the track; thus, they are incapable of real-time detection of the vehicle running state in the entire process. On-board monitoring techniques are increasingly becoming popular in an attempt to improve the quality and efficiency of vehicle condition monitoring and fault diagnosis. If sensors can be installed on the vehicles, the deficiencies of wayside monitoring can be overcome.

Specially adapted wheels are employed to measure wheelrail forces during train motion [7]. The drawback of the instrumented wheel-set is the work volume required for its preparation, including the tedious calibration process and specific design of the instrumented wheels based on different vehicles. Therefore, this technology is hard to generalize. Axle box acceleration signals are a measure of the wheel in the vehicle-track system and are excited during wheel-rail interaction. Therefore, they can indicate irregularities at the wheel-rail interface. Some measurement systems have been designed based on this idea to diagnose track defects [811]. Similarly, axle box acceleration is utilized to automatically detect wheel defects $[12,13]$. A significant advantage of using axle box acceleration measurement is its ease of implementation and maintenance in trains with lower cost. In this paper, we present a detection method for wheel flats based on the measurement of axle box vibration by installed accelerometers. 
However, the vibration signals of axle box are always contaminated by various interferences, such as track irregularity, vehicle speed alteration, and noise. Moreover, many of the vibrations are also excited by normal wheel-rail rolling contact. Therefore, it is a challenging problem to accurately extract the fault relevant characteristic from raw vibration signal in wheel fault diagnosis using axle box acceleration.

The use of intelligent methods to enhance the quality of the signals has been crucial for railway wheel fault diagnosis. Short time Fourier transform and wavelet transform are employed in $[12,13]$. However, both of the methods possess common theoretical flaws. Regardless of how data change, the same basic functions are used for approximation; when the preselected window or basic function does not match the characteristic of the raw signals, incorrect analysis results may occur. EMD is an adaptive signal analysis method, which does not require predetermined basic function [14]. It can be applied to the extraction of nonlinear and nonstationary characteristic of axle box vibration response. However, mode mixing is a significant drawback of EMD, which implies that either a single IMF consists of signals with dramatically disparate scales or a signal of the same scale appears in different IMF components [15]. Aiming to solve this problem, ensemble EMD (EEMD) is put forward by $\mathrm{Wu}$ and Huang [16] and this technology is widely used in mechanical fault diagnosis $[17,18]$. However, EEMD tolerates the residue noise of the signal reconstruction [19]. Therefore, in the present paper, an energy conservation principle of EMD and an IMF superposition principle are proposed for the purpose of eliminating the mode mixing problem and extracting the potential fault information of wheel flat.

The rest of this paper is organized as follows. Section 2 proposes energy conservation principle and IMF superposition principle to eliminate mode mixing of EMD. The improved EMD method and the advantages of it over the EEMD are demonstrated in Section 3. Sections 4 and 5 apply the improved EMD method to wheel flat detection in simulated vehicle operation situations and the test rig experimental situation, respectively. Conclusions are given in Section 6 .

\section{A Mode Mixing Eliminating Method}

In order to eliminate mode mixing phenomenon, an energy conservation principle of EMD and an IMF superposition principle are proposed and derived.

2.1. EMD Energy Conservation Principle. For a time series $x(t)$, the local maxima are connected by a cubic spline as the upper envelope $u(t)$ and the local minima are similarly connected as the lower envelop $v(t)$. The local mean $m(t)$ of the two envelopes is

$$
m(t)=\frac{[u(t)+v(t)]}{2} .
$$

The difference between $x(t)$ and $m(t)$ is given by

$$
h_{1}(t)=x(t)-m(t)
$$

$h_{1}(t)$ is approximately the first IMF. However, it may not meet the requirements of IMFs [14]. Therefore, $h_{1}(t)$ has to be processed again as a new time series as (1) and (2). The new mean $m_{1}(t)$ and the difference $h_{2}(t)$ are

$$
\begin{aligned}
& m_{1}(t)=\frac{\left[u_{1}(t)+v_{1}(t)\right]}{2} \\
& h_{2}(t)=h_{1}(t)-m_{1}(t) .
\end{aligned}
$$

$h_{2}(t)$ is again treated as a new time series, and the process, referred to as "sifting," is repeated many times until the number of zero crossings of $h_{i}(t)$ is equal to the number of extrema or different at most 1 . The first $\operatorname{IMF} c_{1}(t)$ is designated as

$$
c_{1}(t)=h_{i}(t) \text {. }
$$

Once $c_{1}(t)$ is determined, the residue $r_{1}(t)$ can be obtained by separating $c_{1}(t)$ from the raw time series; that is,

$$
r_{1}(t)=x(t)-c_{1}(t)
$$

By taking the residue $r_{1}(t)$ as a new data and repeating the above steps, the second IMF component is obtained:

$$
r_{2}(t)=r_{1}(t)-c_{2}(t)=x(t)-c_{1}(t)-c_{2}(t) .
$$

If $c_{i}(t)$ or $r_{i}(t)$ is smaller than a predetermined value or $r_{i}(t)$ becomes a monotone function, the sifting process is stopped. A series of IMFs can be obtained:

$$
x(t)=\sum_{i=1}^{n-1} c_{i}(t)+r(t) .
$$

The IMFs $c_{i}(t)$ are nearly monocomponent signals in theory, which range from high frequency to low frequency. Based on the completeness and orthogonality theory of EMD [14], the energy of the signal $x(t)$ is constant before and after decomposition in theory, which can be expressed as follows:

$$
\begin{aligned}
E_{x}= & \int_{-\infty}^{\infty} x^{2}(t) d t=\int_{-\infty}^{\infty}\left[\sum_{i=1}^{n-1} c_{i}(t)+r(t)\right]^{2} d t \\
= & \int_{-\infty}^{\infty} c_{1}^{2}(t) d t+\int_{-\infty}^{\infty} c_{2}^{2}(t) d t+\cdots \\
& +\int_{-\infty}^{\infty} c_{n-1}^{2}(t)+\int_{-\infty}^{\infty} r^{2}(t) d t \\
= & E_{1}+E_{2}+\cdots+E_{n-1}+E_{n} .
\end{aligned}
$$


In (8), if IMF $c_{k}(t)$ obtained by EMD is not a real mode component, then, after removing $c_{k}(t)$ from $x(t)$, the total energy $E_{y}$ becomes

$$
\begin{aligned}
E_{y}= & \int_{-\infty}^{\infty} c_{k}^{2}(t) d t+\int_{-\infty}^{\infty}\left[x(t)-c_{k}(t)\right]^{2} d t \\
= & \int_{-\infty}^{\infty} c_{k}^{2}(t) d t+\int_{-\infty}^{\infty} x^{2}(t) d t \\
& -2 \int_{-\infty}^{\infty} x(t) c_{k}(t) d t+\int_{-\infty}^{\infty} c_{k}^{2}(t) d t \\
= & 2 E_{c_{k}}+E_{x}-2 \int_{-\infty}^{\infty} x(t) c_{k}(t) d t .
\end{aligned}
$$

Comparing with (8) and (9), the signal energy may change if an IMF is nonorthogonal. To check the orthogonality of the IMFs, Huang et al. [14] form the square of signal $x(t)$ as

$$
x^{2}(t)=\sum_{j=1}^{n} c_{j}^{2}(t)+2 \sum_{j=1}^{n} \sum_{k=1}^{n} c_{j}(t) c_{k}(t)
$$

in which we have included $r(t)$ in (7) as an additional element. If the decomposition is orthogonal, the cross terms given in the second part on the right-hand side should be zero. In reality, on account of the finite data length, some IMFs are not orthogonal. Suppose that $d_{k}(t)$ is the orthogonal component in theory. $e_{k}(t)$ is the error between $d_{k}(t)$ and $c_{k}(t)$ :

$$
d_{k}(t)=c_{k}(t)+e_{k}(t) \text {. }
$$

Substitute (11) into (9):

$$
\begin{aligned}
E_{y}= & 2 E_{c_{k}}+E_{x}-2 \int_{-\infty}^{\infty} x(t) c_{k}(t) d t \\
= & 2 E_{c_{k}}+E_{x}-2 \int_{-\infty}^{\infty} x(t)\left[d_{k}(t)-e_{k}(t)\right] d t \\
= & 2 E_{c_{k}}+E_{x}-2 \int_{-\infty}^{\infty} x(t) d_{k}(t) d t \\
= & 2 E_{c_{k}}+E_{x} \\
& -2 \int_{-\infty}^{\infty}\left[c_{1}(t)+c_{2}(t)+\cdots+c_{n}(t)\right] d_{k}(t) d t \\
= & 2 E_{c_{k}}+E_{x}-2 \int_{-\infty}^{\infty} c_{k}(t) d_{k}(t) d t \\
= & 2 E_{c_{k}}+E_{x}-2 \int_{-\infty}^{\infty}\left[d_{k}(t)-e_{k}(t)\right] d_{k}(t) d t \\
= & 2 E_{c_{k}}+E_{x}-2 \int_{-\infty}^{\infty} d_{k}^{2}(t) d t \\
= & 2 \int_{-\infty}^{\infty}\left[d_{k}(t)-e_{k}(t)\right]^{2} d t+E_{x} \\
& -2 \int_{-\infty}^{\infty} d_{k}^{2}(t) d t=E_{x}+2 E_{e_{k}} .
\end{aligned}
$$

In the derived process of (12), $x(t)$ and $e_{k}(t)$ are nonorthogonal; thus $\int_{-\infty}^{\infty} x(t) e_{k}(t)=0$. Therefore, the third row in (12) can be obtained from the second row. Analysis for other components is similar.

Equation (12) shows that the signal energy remains unchanged before and after decomposition when the decomposition results are all orthogonal components; namely, at this time, $e(t)=0, E_{e_{k}}=0$, and $E_{y}=E_{x}$. If some of the decomposition results are not orthogonal components, the decomposition results disobey the energy conservation principle and the energy increases after decomposition; namely, at this time, $e(t) \neq 0, E_{e_{k}}>0$, and $E_{y}>E_{x}$.

According to the above analysis, signal energy can be used as a criterion to evaluate the decomposition effectiveness of EMD.

2.2. IMF Superposition Principle. Some of the IMFs obtained by EMD are false mode components. In order to eliminate those IMFs and reserve intrinsic mode components, the property of false mode components is illustrated. Here a typical simulated signal $y(t)=\cos (2 \pi 220 t)$ is employed. The sampling frequency of signal $y(t)$ is $1024 \mathrm{~Hz}$ and the sampling number is 500. EMD decomposition results and their corresponding Fast Fourier Transform (FFT) spectrum are shown in Figures 1 and 2, respectively.

There should be only one IMF obtained after EMD decomposition in theory, while two IMFs emerge in Figure 1. As a result, EMD decomposition produces a false mode component (IMF2) and IMF1 has been polluted at the same time. The above decomposition process can be described as

$$
y(t)=\operatorname{imf}_{1}(t)+\operatorname{imf}_{2}(t) .
$$

IMF1 contains raw signal $y(t)$ and decomposition error $e(t)$; IMF2 is a false mode component, which can be expressed as $f(t)$. Therefore, (13) can be written as

$$
y(t)=y(t)+e(t)+f(t)
$$

From (14) we know that the amplitude of the false mode component equals the decomposition errors hidden in the IMFs, but the direction is opposite:

$$
e(t)=-f(t) \text {. }
$$

The energy of raw signal $y(t)$ equals

$$
\begin{aligned}
E_{r} & =\int_{-\infty}^{\infty} y^{2}(t) d t=\int_{-\infty}^{\infty}\left[\operatorname{imf}_{1}(t)+\operatorname{imf}_{2}(t)\right]^{2} d t \\
& =\int_{-\infty}^{\infty}\left[\operatorname{imf}_{1}^{2}(t)+\operatorname{imf}_{2}^{2}(t)\right. \\
& \left.+2 \operatorname{imf}_{1}(t) \operatorname{imf}_{2}(t)\right] d t=\int_{-\infty}^{\infty}\left[\operatorname{imf}_{1}^{2}(t)\right. \\
& \left.+\operatorname{imf}_{2}^{2}(t)+2(y(t)-f(t)) f(t)\right] d t \\
& =\int_{-\infty}^{\infty}\left[\operatorname{imf}_{1}^{2}(t)+\operatorname{imf}_{2}^{2}(t)-2 f^{2}(t)\right] d t .
\end{aligned}
$$




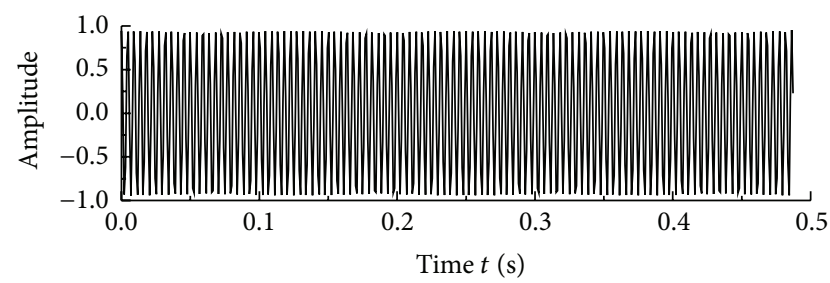

(a) IMF1

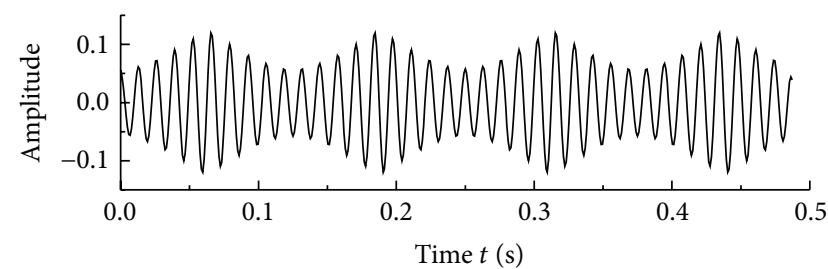

(b) IMF2

FIGURE 1: EMD decomposition results of $y(t)$.

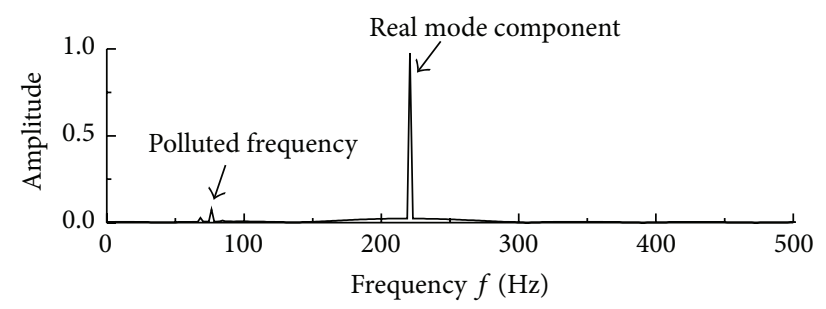

(a) IMF1

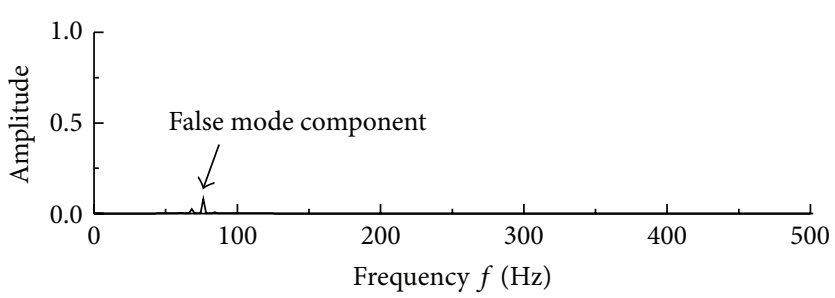

(b) IMF2

FIGURE 2: FFT spectrum of the signal presented in Figure 1.

The energy of the signal after decomposition is

$$
E_{d}=\int_{-\infty}^{\infty}\left[\operatorname{imf}_{1}^{2}(t)+\operatorname{imf}_{2}^{2}(t)\right] d t
$$

In (16), $\operatorname{imf}_{1}(t)$ is a real mode component, whereas $\operatorname{imf}_{2}(t)$ is a false mode component. When the real mode component $\operatorname{imf}_{1}(t)$ is added to the false mode component $\operatorname{imf}_{2}(t)$, the total energy $E_{r}$ of $\operatorname{imf}_{1}(t)+\operatorname{imf}_{2}(t)$ would be less than the sum of the energy $E_{d}$ of $\operatorname{imf}_{1}(t)$ and $\operatorname{imf}_{2}(t)$. At this time, $f(t)>0$. That is to say, if a false mode component is added to a real mode component, the energy of this new signal would be less than the sum of false mode component's energy and real mode component's energy.

Therefore, according to this property, the false mode component can be picked out easily. What is more, from (16) and (17), we know that $E_{r}<E_{d}$, which is consistent with the viewpoint in Section 2.1.

2.3. The Procedure of Removing False Mode Components. This paper presents a novel approach that detects and eliminates false mode components based on Sections 2.1 and 2.2. The proposed approach is summarized as follows.

Step 1. Detect the IMFs according to energy conservation principle. If $E_{x}^{2}<\sum_{i=1}^{n} E_{x_{i}}^{2}$, which means part of IMFs are nonorthogonal and false mode components exist, the decomposition results should be checked further.

Step 2. The cross-correlation coefficient between each IMF and the original signal is calculated. The IMF $c_{i}(t)$ with the maximum cross-correlation coefficient is selected as a real mode component.

Step 3. If the $j$ th mode component $c_{j}(t)$ is added to $c_{i}(t)$ in the time domain, the total energy decreases; that is,
$E_{c_{i}+c_{j}}^{2}<E_{c_{i}}^{2}+E_{c_{j}}^{2}$, where $c_{j}(t)$ is judged as a false mode component. Otherwise, $c_{j}(t)$ is judged as a real mode component.

Step 4. False mode components are subtracted from the original signal. And then conduct EMD again.

Stop Criterion. All false mode components have been eliminated.

\section{The Improved EMD Method and Validation}

3.1. The Improved EMD Method. Based on the research findings in Section 2, the work steps of improved EMD method are depicted in Figure 3.

Firstly, mathematical morphology filtering is conducted for the purpose of eliminating noises and interferences. Mathematical morphology filtering is a nonlinear signal processing technique based on set theory [20]. The main idea of morphology filtering can be described as follows: it treats signal as a set and selects a smaller set called structure element to modify signal geometric shape for removing noise and extracting useful information [21].

Secondly, the filtered signal is decomposed by EMD. Furthermore, the false mode components are eliminated by the method proposed in Section 2.3. After this operation, the IMFs are all real mode components, which can greatly clean the signal and highlight fault characteristic more clearly.

Next, an IMF which is more sensitive to fault characteristic is selected according to [18]. The details of the selection process proposed in [18] are summarized as follows:

(1) Calculate the correlation coefficient $u_{n}$ between the $n$th IMF and the filtered results of a faulty signal $s_{f}(t)$. 


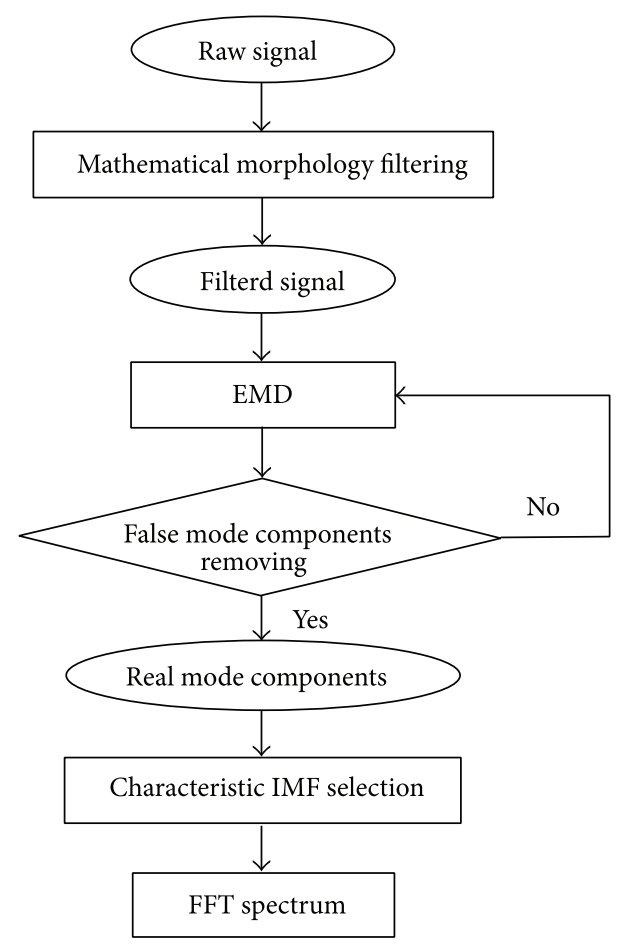

FIGURE 3: Flow chart of the improved EMD method.

(2) Calculate the correlation coefficient $\beta_{n}$ between the $n$th IMF and the filtered results of a normal signal $s_{n}(t)$.

(3) Define and calculate the fault-related coefficient $\eta_{n}$ by combining the above two kinds of correlation coefficients $u_{n}$ and $\beta_{n}$ :

$$
\eta_{n}=u_{n}-\beta_{n}
$$

(4) Define and calculate the sensitivity factor $\lambda_{n}$ of IMFs of the signal $s_{f}(t)$ by normalizing $\eta_{n}$ according to the following equation:

$$
\lambda_{n}=\frac{\eta_{n}-\min (\eta)}{\max (\eta)-\min (\eta)}, \quad \eta=\left\{\eta_{n}\right\} .
$$

(5) Rank all the IMFs of the signal $s_{f}(t)$ in terms of their sensitivity factors from large to small; the IMF with the biggest sensitivity factor is selected as sensitive IMF.

Finally, FFT spectrum of the sensitive IMF selected is obtained. The fault can be detected based on the characteristic frequencies in FFT spectrum.

3.2. Method Verification. A simulated signal $z(t)$ is formulated as follows to verify the advantage of the improved EMD method:

$$
z(t)=z_{1}(t)+z_{2}(t)+z_{3}(t)
$$

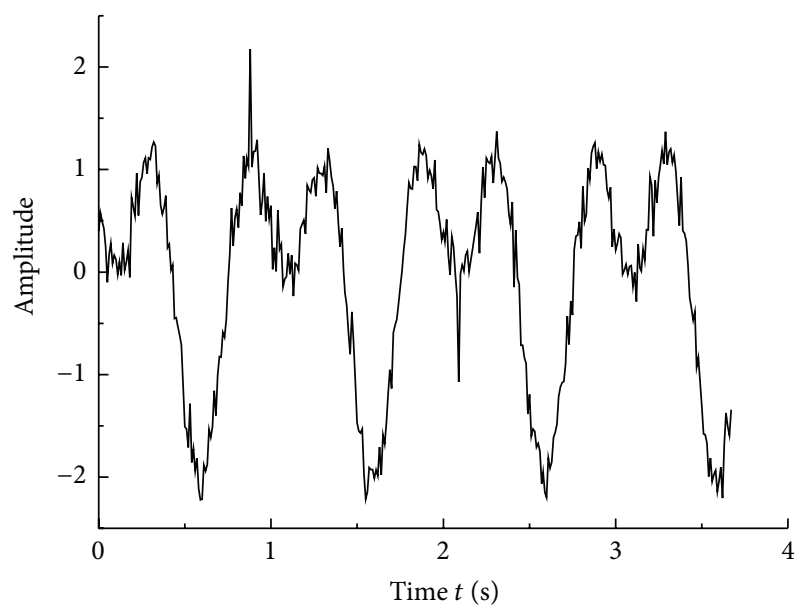

FIgURE 4: The simulated signal $z(t)$.

where $z_{1}(t)$ is the sum of two harmonic waves $z_{1}(t)=$ $\sin (2 \pi t)+\cos (4 \pi t) ; z_{2}(t)$ is a pulse interference with amplitude 1 in time $0.88 \mathrm{~s}$ and $2.10 \mathrm{~s} ; z_{3}(t)$ is the Gaussian noise. The signal-to-noise ratio (SNR) of signal $z(t)$ is $16 \mathrm{~dB}$, and the sample frequency is $100 \mathrm{~Hz}$. The time evolution of this simulated signal is shown in Figure 4.

The synthesized signal is processed by a multiscale morphological filter with a flat structure element. The denoised signal is then decomposed using EMD and the IMFs are shown in Figure 5, from which the mode mixing still exists in the IMFs. The cause of mode mixing mainly comes from the pulse signal of $z_{2}(t)$, which greatly changes the distribution of extreme points.

On the basis of IMF superposition principle, we find that IMF1 and IMF6 in Figure 5 are false mode components. Subtract them from the filtered signal and execute EMD again; the results are shown in Figure 6. It reflects that the two harmonic components contained in the original signal are decomposed into two IMFs perfectly, where the third IMF denotes the $2 \mathrm{~Hz}$ cosine signal and the fourth IMF indicates the $1 \mathrm{~Hz}$ sine signal.

Different from the practical application in railway wheel flat detection, a vibration signal with a healthy wheel exists; the example in Section 3.2 does not have a reference signal; thus the penultimate step in the flow chart of improved EMD method presented in Figure 3 is ignored. IMF3 and IMF4 in Figure 6 are selected as the characteristic IMFs according to the prior knowledge and their Hilbert spectrums are shown in Figure 7. From Figure 7, the characteristic frequencies of $2 \mathrm{~Hz}$ and $1 \mathrm{~Hz}$ are clearly detected. Therefore, the improved EMD method is able to solve mode mixing problem and achieves an improved decomposition.

EEMD is employed to analyze the simulated signal $z(t)$ for comparing. Firstly, the recommended parameters of EEMD [16] are used: the ensemble number is 100; the standard deviation of the added noise is 0.2 times the standard deviation of the raw signal. Quite contrary to what is expected, IMFs do not imitate the two harmonic components of synthesized signal. The mode mixing is occurring. Due to space limitation, this result is not shown. 

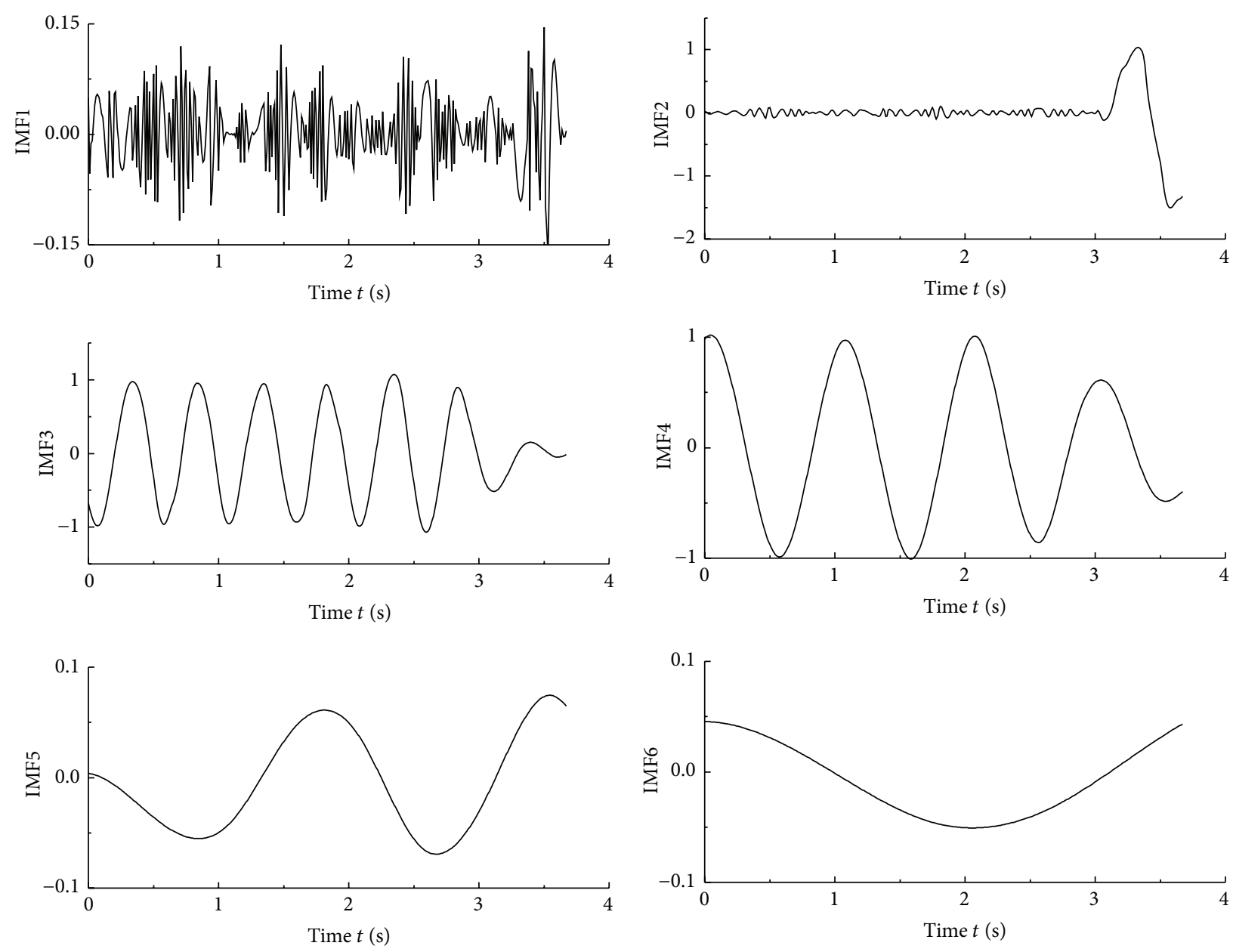

FIGURE 5: EMD decomposition results of the filtered signal.

Zhang et al. [22] pointed out that EEMD would present a better result when the SNR between the raw signal and the noise added is within the range of 50-60 dB. Therefore, the simulation signal in Figure 4 is decomposed using EEMD with the ensemble number 100 and the standard deviation of the added noise changes into 0.0017 times the standard deviation of the raw signal $z(t)(\mathrm{SNR}=54.75 \mathrm{~dB})$. Figure 8 shows the first six IMFs extracted using this procedure. It can be seen that the mode mixing phenomenon still exists. The characteristic frequencies of $2 \mathrm{~Hz}$ and $1 \mathrm{~Hz}$ cannot be detected accurately. Correspondingly, the two harmonic frequencies are partly lost from the Hilbert spectrum shown in Figure 9.

From Figures 6-9 we can conclude that the improved EMD method provides better performance than EEMD for analyzing the simulated signal $z(t)$ presented in (20). What is more, the effectiveness of EEMD greatly relies on the parameter selection and the SNR criterion proposed by Zhang et al. [22] may not be proper for analyzing all signals $[23,24]$. But the improved EMD, with no parameters needed to be defined beforehand, is totally adaptive.

\section{Simulated Model Analysis}

4.1. Vehicle-Track Coupling Dynamics Model. A model has been developed based on multibody dynamics theory, which includes the lateral, vertical, and longitudinal coupling dynamics of vehicle and track. The model includes 1 car body, 2 bogie frames, 8 axle boxes, and 4 wheel-sets. Both the car body and bogie frame have 6 degrees of freedom, whereas the axle box only has 1 in the vertical direction. One wheel-set has 6 degrees of freedom, in which the rolling and vertical degrees of freedom are dependent. The system has 15 rigid bodies, 42 independent degrees of freedom, and 8 dependent degrees of freedom. The nonlinear relationship of the wheel-rail contact is considered. Because only the vertical vibration of axle box is used for fault diagnosis of wheel flat, here the vehicle system dynamic equation is written as follows:

$$
M \ddot{q}+C \dot{q}+K q=f(\ddot{q}, q, t)+G e,
$$

where $M, C$, and $K$ are the mass, damping, and stiffness matrices of the vehicle model, respectively; $f$ is the nonlinear force element; $G$ is the input distribution matrix of track excitation; $e$ is track irregularity input; $q, \dot{q}$, and $\ddot{q}$ are vertical displacement, velocity, and acceleration, respectively; and $t$ is time. The details for this model can be seen also in [25].

The wheel flat model in $[26,27]$ is equivalent to the knockon effects of the low-orbit joint; namely, the wheel-rail impact caused by a wheel flat is calculated by treating the wheel flat as a periodic track irregularity, which achieved suitable results. 

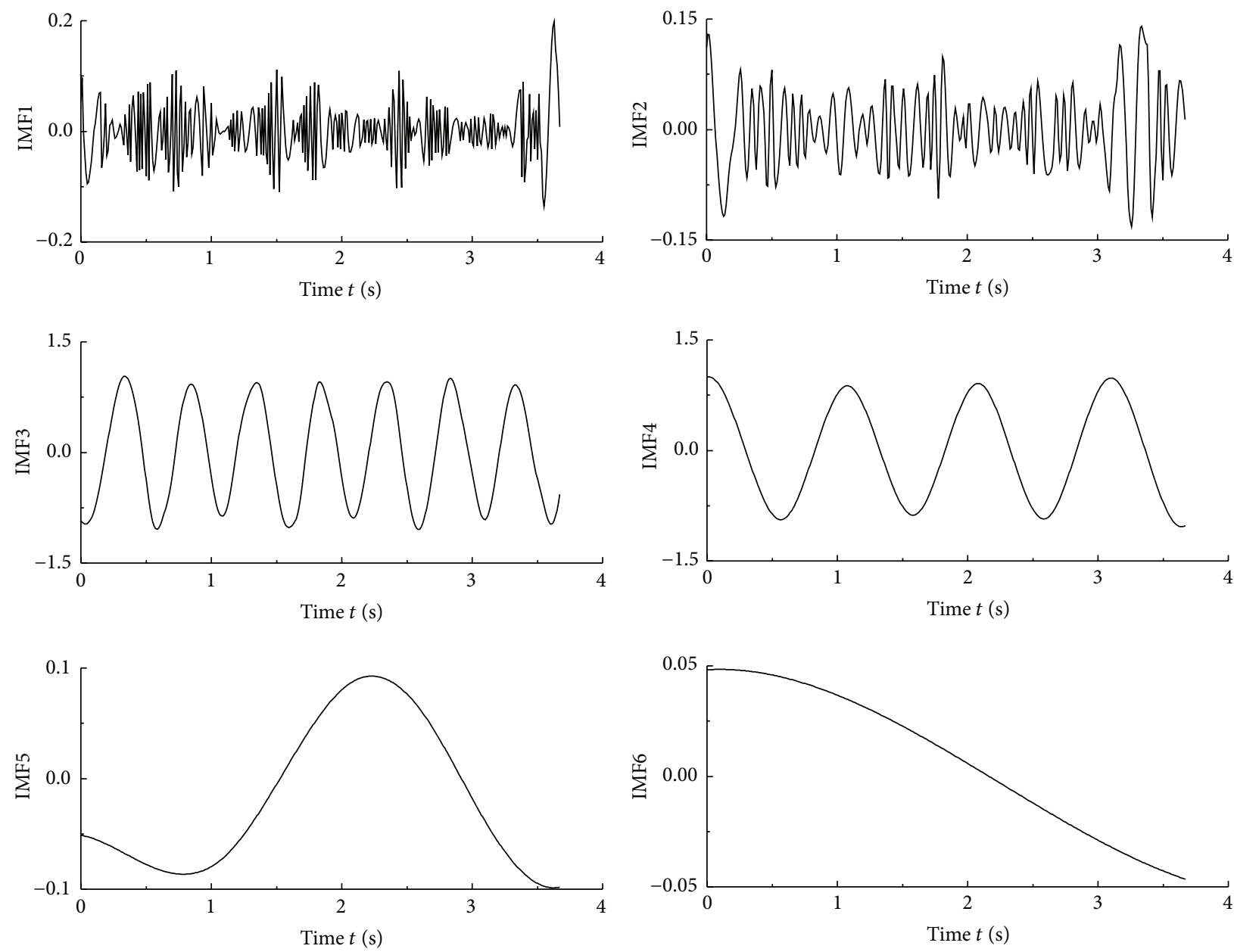

FIGURE 6: Improved EMD analysis results of the simulated signal $z(t)$.

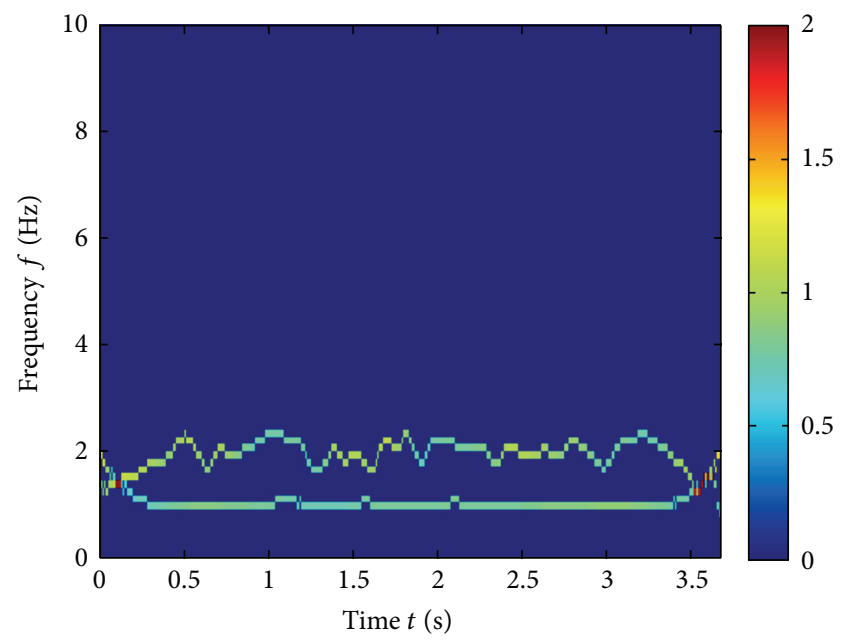

Figure 7: Hilbert spectrum of the simulated signal $z(t)$ after improved EMD analysis.

The same method is adopted in this study to calculate the impact response.
4.2. Detection of Wheel Flat. Axle box vertical vibration response of the vehicle with a flat wheel is calculated and shown in Figure 10. The conditions are as follows: the sample frequency is $1000 \mathrm{~Hz}$; the sampling number is 2000; the wheel flat length is $10 \mathrm{~mm}$; vehicle running speed is $100 \mathrm{~km} / \mathrm{h}$; and the track irregularity is the American fifth-grade track irregularity. The wheel diameter is $0.88 \mathrm{~m}$; thus the fault frequency of wheel flat is $10 \mathrm{~Hz}$.

The improved EMD is employed to process the vibration signal shown in Figure 10. Figure 11 illustrates the characteristic IMF selected and its FFT spectrum. Figure 11(b) suggests that the fault frequency of $10 \mathrm{~Hz}$ is clearly detected. Therefore, the improved EMD method is effective to identify wheel flat fault.

The EEMD analysis results are presented in Figure 12. The standard deviation of the noise added is set to 0.0017 times the standard deviation of the raw signal $(\mathrm{SNR}=53.20 \mathrm{~dB})$. As shown in Figure 12(b), the wheel flat impulsive characteristic frequency of $10 \mathrm{~Hz}$ is detected, but the interference is also severe comparing with Figure 11(b). One reason of EEMD getting acceptable analysis result is that the raw signal in Figure 10 is relatively simpler, as the impulsive features can 

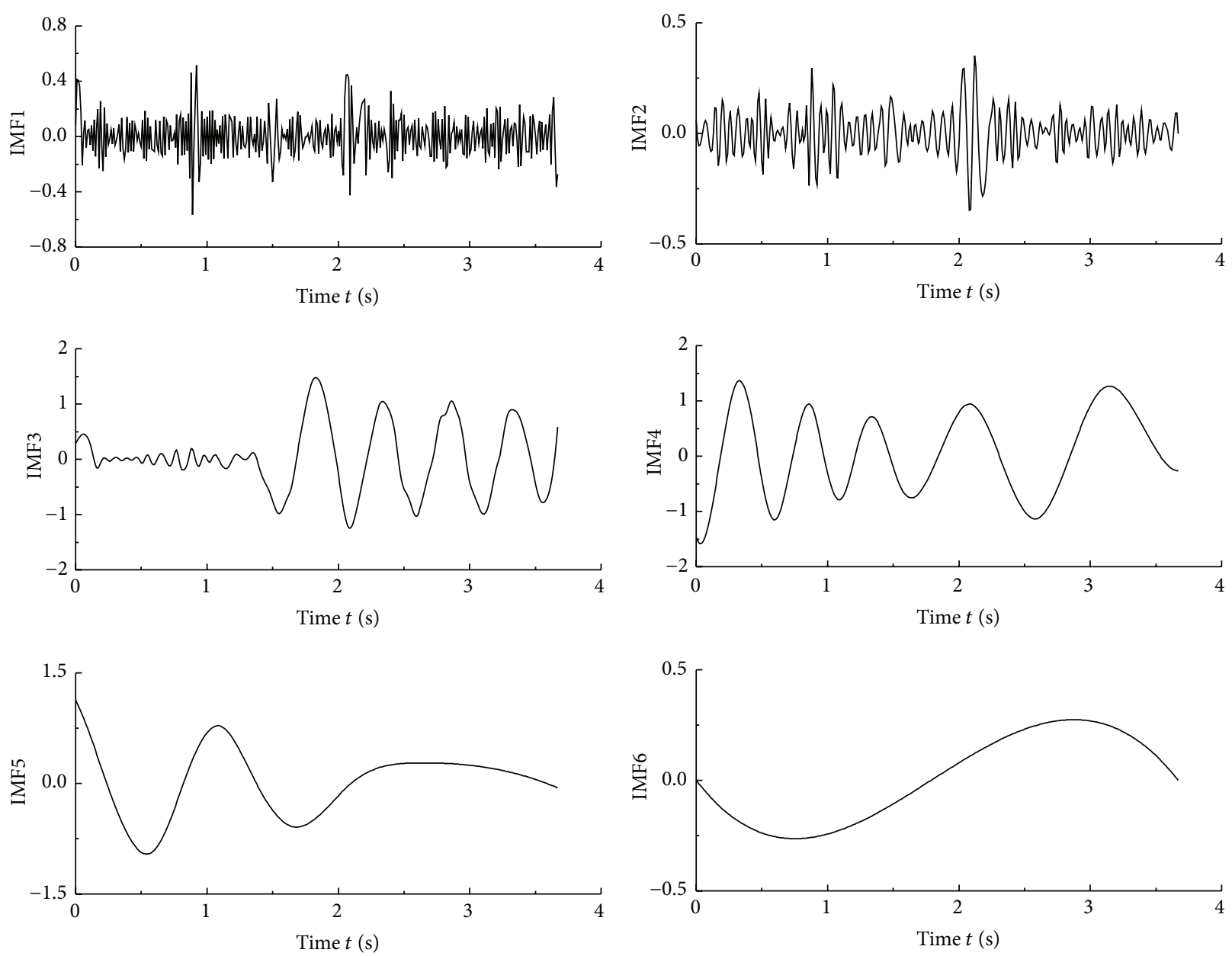

FIGURE 8: EEMD analysis results of the simulated signal $z(t)$.

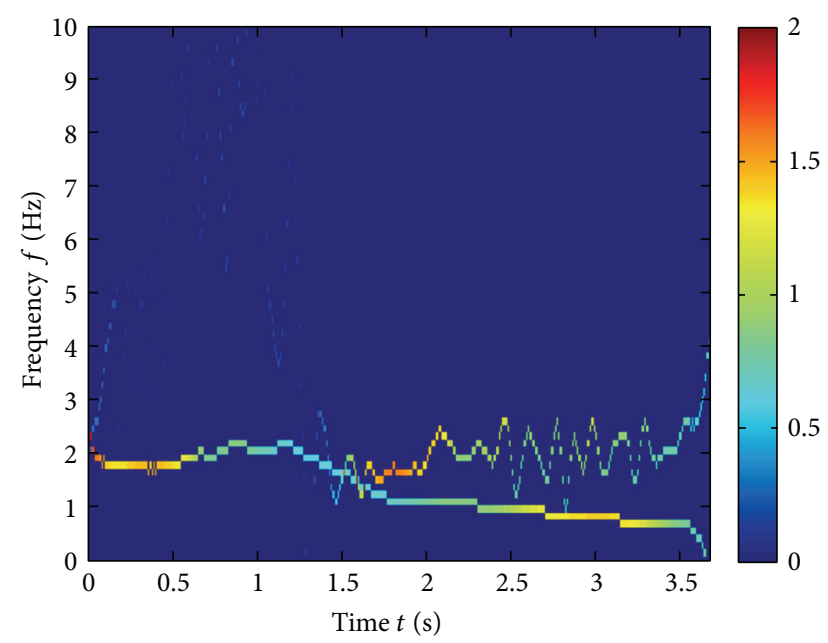

Figure 9: Hilbert spectrum of the simulated signal $z(t)$ after EEMD analysis.

be seen directly in time domain. When the operation circumstance becomes more complicated, the detection ability of EEMD may not be as good as this.

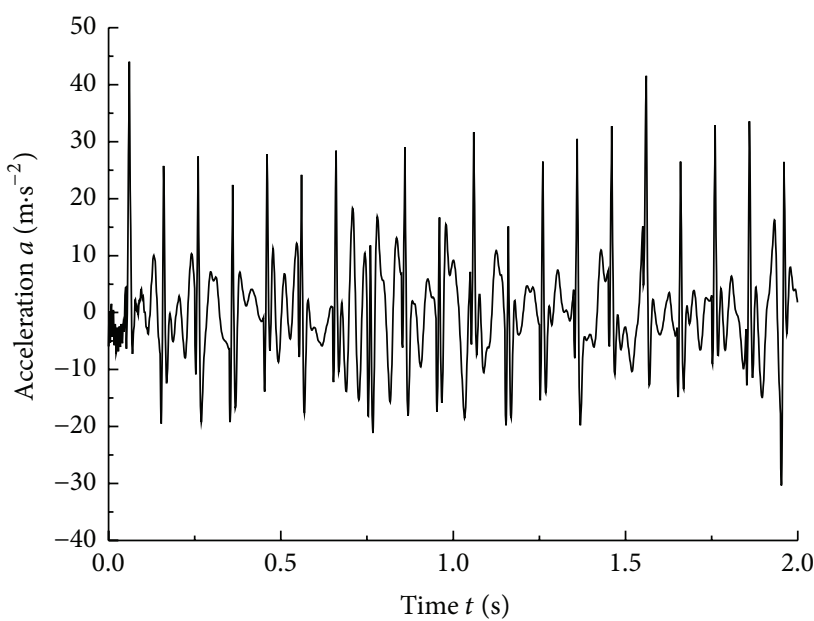

FIGURE 10: The axle box vibration signal.

4.3. Influence of Track Irregularity. Vehicle vibration status is largely influenced by the geometrical parameter of tracks. Different track conditions vary the vibration response of axle box, which disturbs wheel flat detection and diagnosis. This 


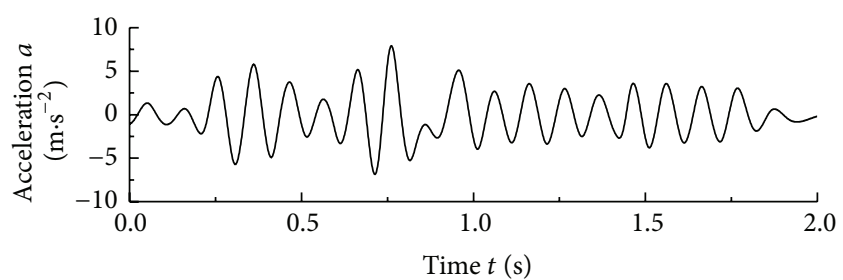

(a) The characteristic IMF selected

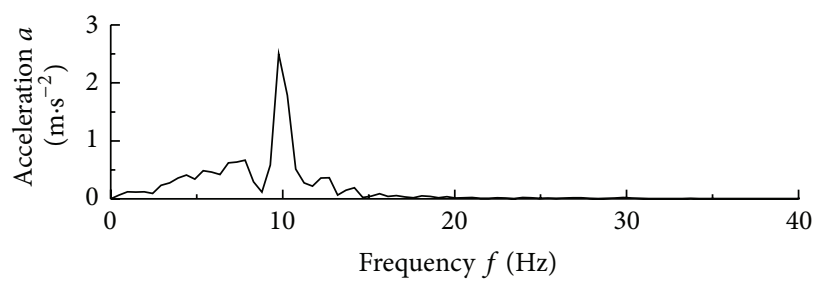

(b) Frequency spectrum

FIGURE 11: Analysis results of the signal presented in Figure 10 by improved EMD: (a) the characteristic IMF and (b) FFT spectrum.

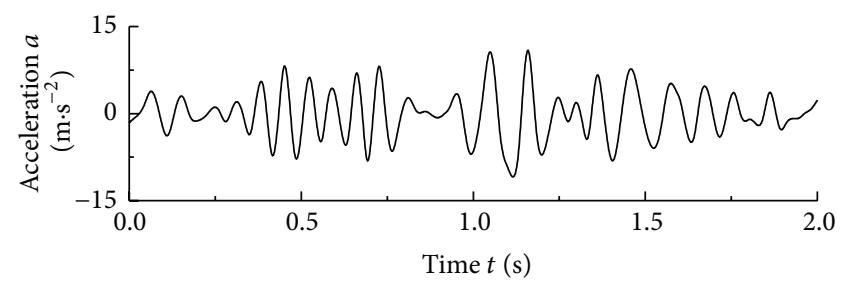

(a) The characteristic IMF selected

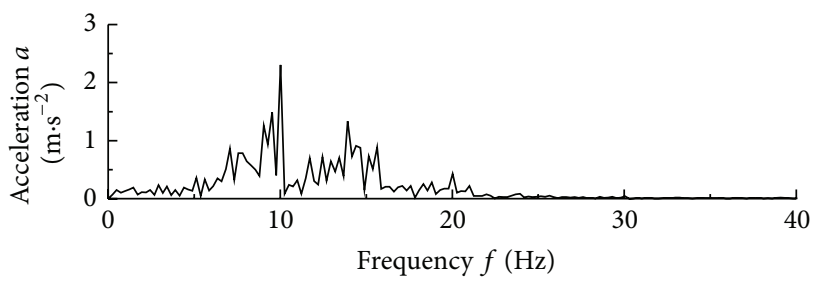

(b) Frequency spectrum

FIGURE 12: Analysis results of the signal presented in Figure 10 by EEMD: (a) the characteristic IMF and (b) FFT spectrum.

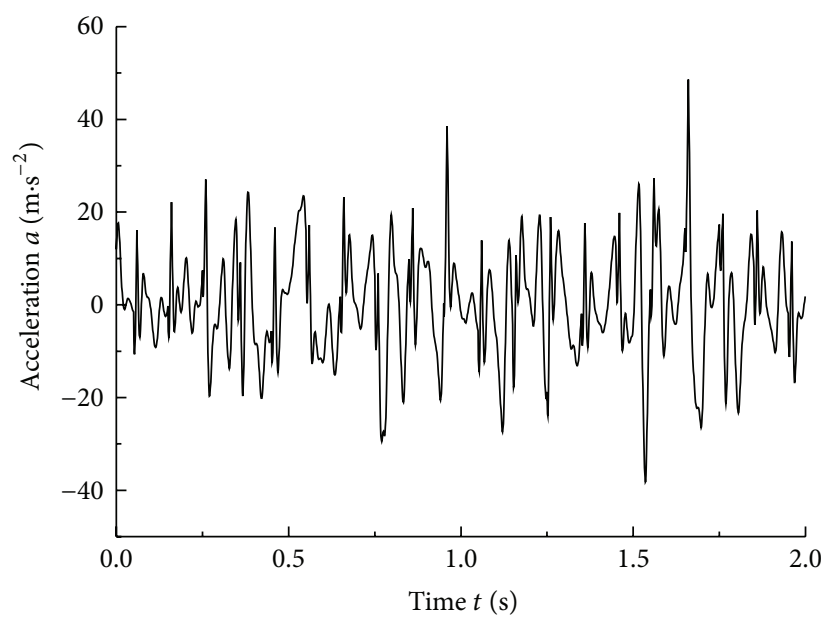

FIGURE 13: The influence of track irregularity.

simulation adopts another track irregularity, the American third-grade track irregularity, for analyzing the influence of track irregularity on wheel flat detection. The other parameters are similar to those in Section 4.2.

Figure 13 shows the time history of the axle box vertical acceleration in this case. Because the track condition of the American third-grade track irregularity is worse than the American fifth-grade track irregularity that is depicted in Figure 10, the characteristic of the flat impact in the time domain is nearly covered.

Figures 14(a) and 14(b) show the time domain and frequency domain representation of the analysis results by improved EMD method, respectively. Figure 14(a) indicates that the proposed method can eliminate mode mixing phenomenon, extract the impact characteristic, and remove the noise. Therefore, the fault frequency of $10 \mathrm{~Hz}$ is clearly presented in Figure 14(b).
The EEMD analysis results are displayed in Figure 15. The standard deviation of the added noise changes to 0.0015 times the standard deviation of the raw signal; at this time the $\mathrm{SNR}=56.33 \mathrm{~dB}$. From Figure 15(a) we can see that the width of each shock is constantly changed and mode mixing still exists, which result in wheel flat fault feature losses of FFT spectrum in Figure 15(b). The wheel flat fault frequency of $10 \mathrm{~Hz}$ cannot be found clearly.

4.4. Influence of Speed. The increase in vehicle running speed aggravates the impact amplitude and frequency of wheel flat. The effect further increases the axle box vibration response. The axle box acceleration is calculated with vehicle running speeds of $200 \mathrm{~km} / \mathrm{h}$ and other parameters similar to those in Section 4.3 to examine the influence of vehicle running speed on identification capability of improved EMD. The time domain vibration signal of axle box is displayed in Figure 16. 


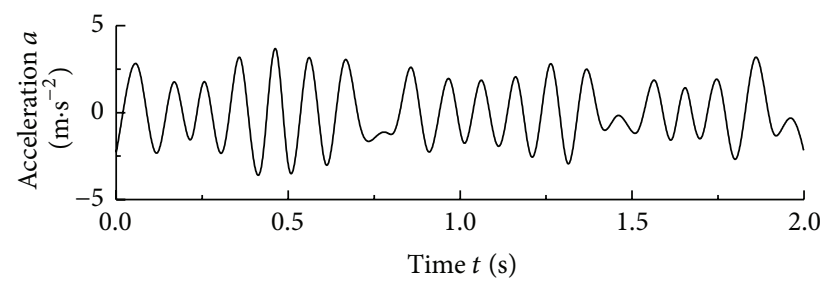

(a) The characteristic IMF selected

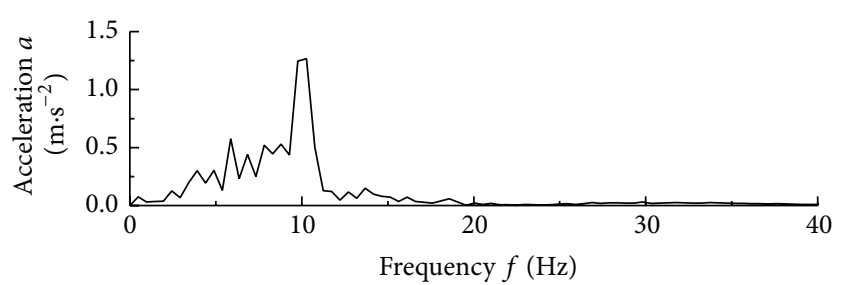

(b) Frequency spectrum

FIGURE 14: Analysis results of the signal presented in Figure 13 by improved EMD: (a) the characteristic IMF and (b) FFT spectrum.

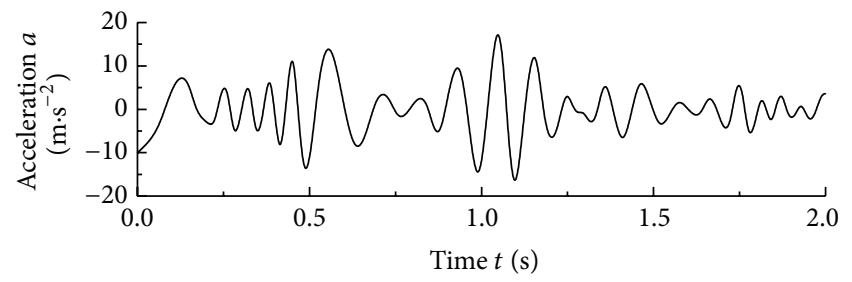

(a) The characteristic IMF selected

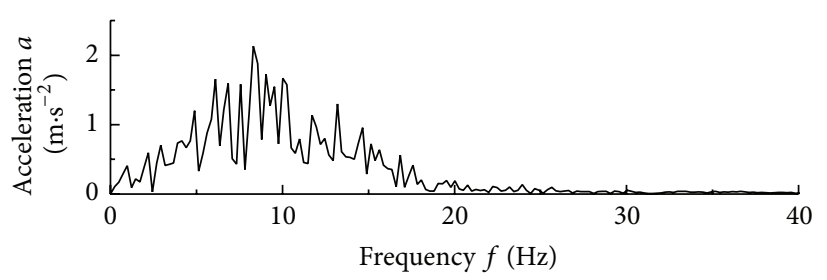

(b) Frequency spectrum

FIGURE 15: Analysis results of the signal presented in Figure 13 by EEMD: (a) the characteristic IMF and (b) FFT spectrum.

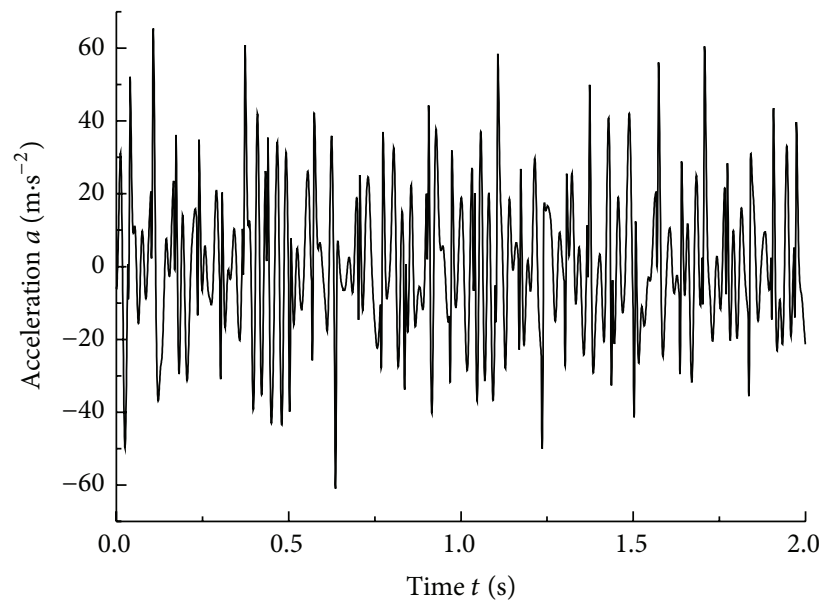

FIGURE 16: The influence of speed.

The sample frequency is $2000 \mathrm{~Hz}$ and the sampling number is 4000 . The wheel flat fault frequency is $20 \mathrm{~Hz}$ in theory on account of the increase of speed.

Figure 17 illustrates the characteristic IMF selected by improved EMD and its FFT spectrum. Based on Figure 17(b), the impulsive frequency of $20 \mathrm{~Hz}$ is clearly detected. Therefore, improved EMD can identify wheel flat fault with variations in speed accurately.

The EEMD process results are shown in Figure 18. The standard deviation of the noise added is 0.0019 times the standard deviation of the raw signal; at this time the SNR = $54.56 \mathrm{~dB}$. From Figure 18(b) we can see that although the fault frequency $20 \mathrm{~Hz}$ is roughly detected, it is heavily contaminated by interferences.

\section{Test Rig Experiment}

To validate the practicability of the proposed improved EMD, a vehicle bench test is performed on the roller test rig.
5.1. Experimental Setup. The rolling stock field simulator is shown in Figure 19. This field simulator can conduct bench tests simulating on-track running conditions with high accuracy and also can be used to examine signs of faults that are difficult to exam by test on tracks by setting faults in bench tests. A single vehicle is set on rollers, which acts as rail in the field simulator. The roller motion is provided by servo hydraulic actuators; these actuators are controlled by a digital controller which allows the inputs to follow defined waveforms or measured track irregularity.

As indicated in Figure 19, an acceleration sensor is installed on axle box. To reduce costs, a rubber block is attached to the surface of the roller to simulate the impact of wheel flat. The diameter of the roller is $1.8 \mathrm{~m}$.

5.2. Experimental Results. Figure 20 presents the time history of the axle box vertical acceleration obtained through onsite measurement. The vehicle running speed is $40 \mathrm{~km} / \mathrm{h}$. The theoretically calculated wheel flat fault frequency is $2 \mathrm{~Hz}$. 


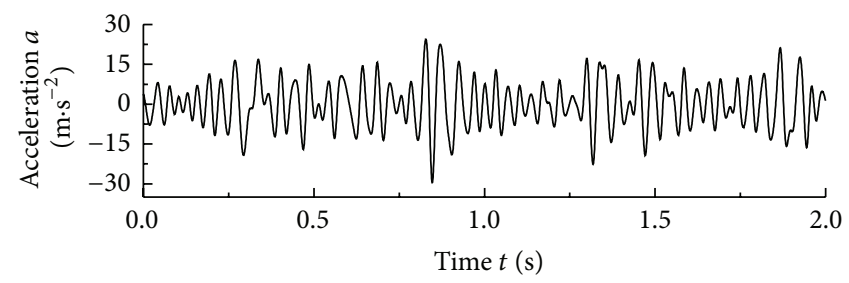

(a) The characteristic IMF selected

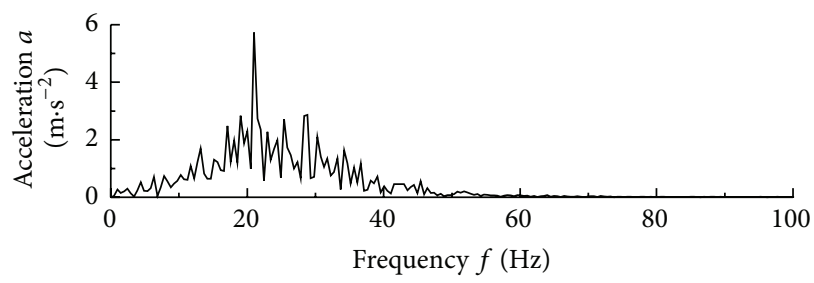

(b) Frequency spectrum

FIGURE 17: Analysis results of the signal presented in Figure 16 by improved EMD: (a) the characteristic IMF and (b) FFT spectrum.

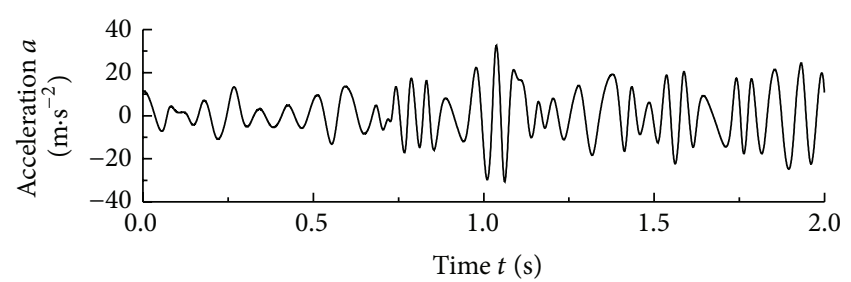

(a) The characteristic IMF selected

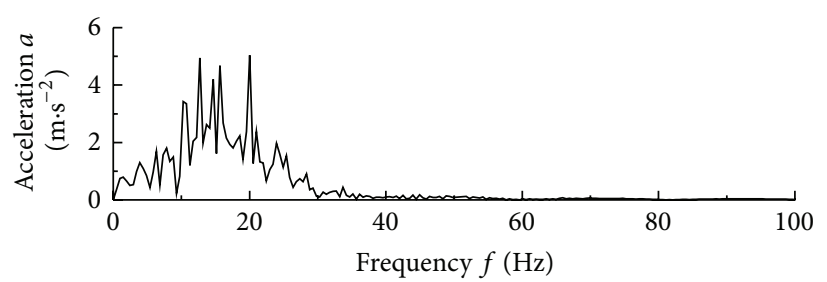

(b) Frequency spectrum

FIGURE 18: Analysis results of the signal presented in Figure 16 by EEMD: (a) the characteristic IMF and (b) FFT spectrum.
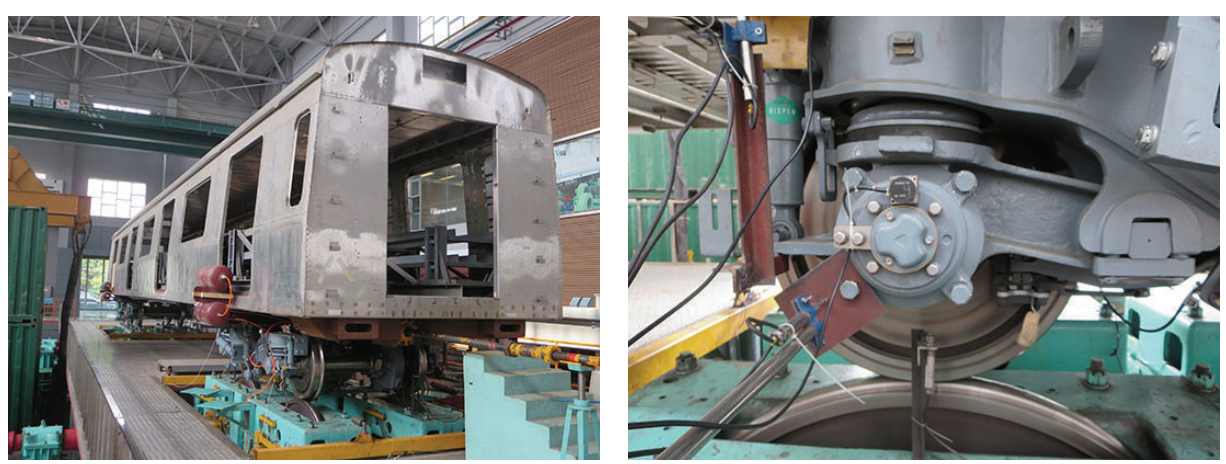

FIGURE 19: Experimental setup.

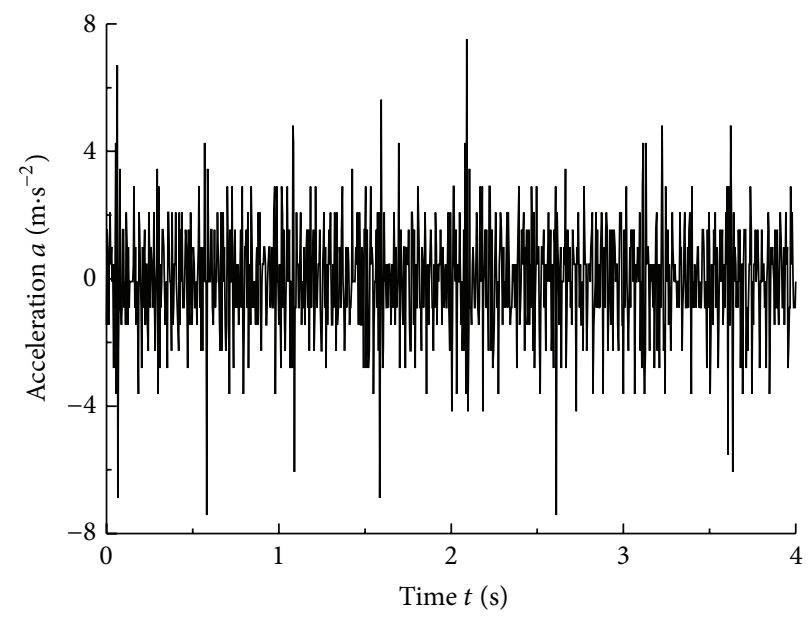

FIGURE 20: Vibration waveform of test rig experiment at the speed of $40 \mathrm{~km} / \mathrm{h}$. 


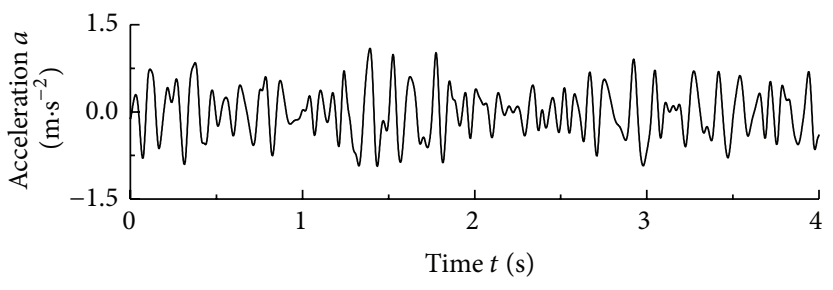

(a) The characteristic IMF selected

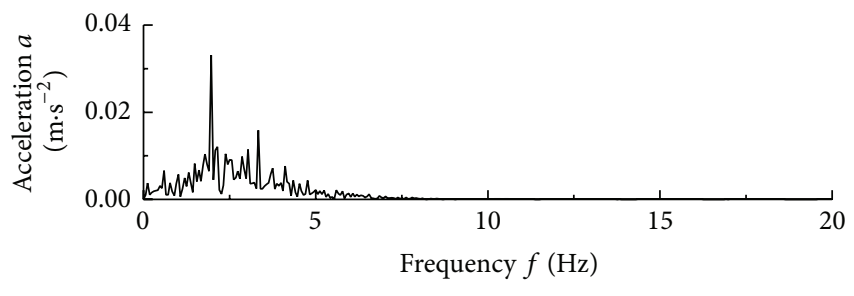

(b) Frequency spectrum

FIGURE 21: Analysis results of the signal presented in Figure 20 by improved EMD: (a) the characteristic IMF and (b) FFT spectrum.

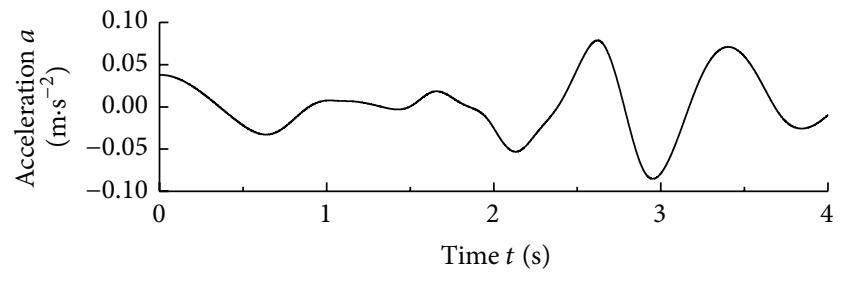

(a) The characteristic IMF selected

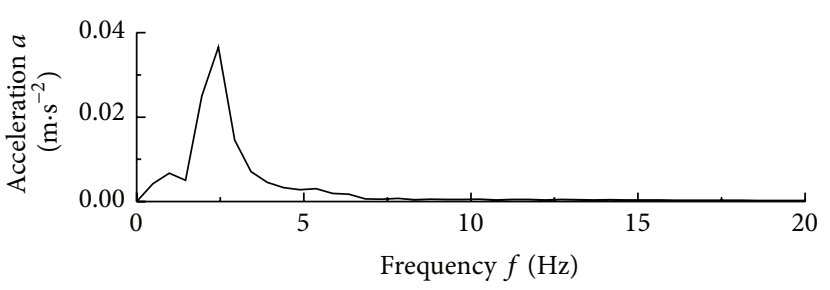

(b) Frequency spectrum

FIGURE 22: Analysis results of the signal presented in Figure 20 by EEMD: (a) the characteristic IMF and (b) FFT spectrum.

The analysis results of the signal presented in Figure 20 are shown in Figure 21 by using improved EMD. There is a favorable match between the estimated features and the actual fault features associated with the railway wheel with flat. This indicates that the proposed method is effective to detect wheel flat fault.

The EEMD analysis results are shown in Figure 22. The standard deviation of the noise added is 0.0019 times the standard deviation of the raw signal $(\mathrm{SNR}=55.66 \mathrm{~dB})$. The fault frequency $2 \mathrm{~Hz}$ could be detected in the Fourier spectrum. However, this dominating spectral line in Figure 22(b) has nothing to do with the actual wheel fault. The time domain curve in Figure 22(a) is most likely EEMD decomposition residue rather than the periodic impulse caused by wheel flat. The presence of a $2 \mathrm{~Hz}$ frequency component in Figure 22(b) is just a coincidence.

Another experiment is conducted in this test rig at the speed of $100 \mathrm{~km} / \mathrm{h}$. The theoretically calculated wheel flat fault frequency is $4.9 \mathrm{~Hz}$. Figure 23 presents the time history and spectrum of the axle box vertical acceleration in this situation.

Figure 24 depicts the analysis results generated by applying the proposed EMD and its spectrum. From Figure 24(b), a fault frequency of $4.9 \mathrm{~Hz}$ is clearly detected. Therefore, the effectiveness of the proposed algorithm is validated.

Figure 25 shows the results produced by EEMD. The standard deviation of the noise added is 0.0016 times the standard deviation of the raw signal; at this time SNR = $55.95 \mathrm{~dB}$. From Figure 25(b), the fault characteristic of wheel flat cannot be detected.

In summary of Figures 20-25, with the help of the false mode removing of EMD, the improved EMD algorithm leads to better performance in terms of fault feature extracting, as well as interference reduction.

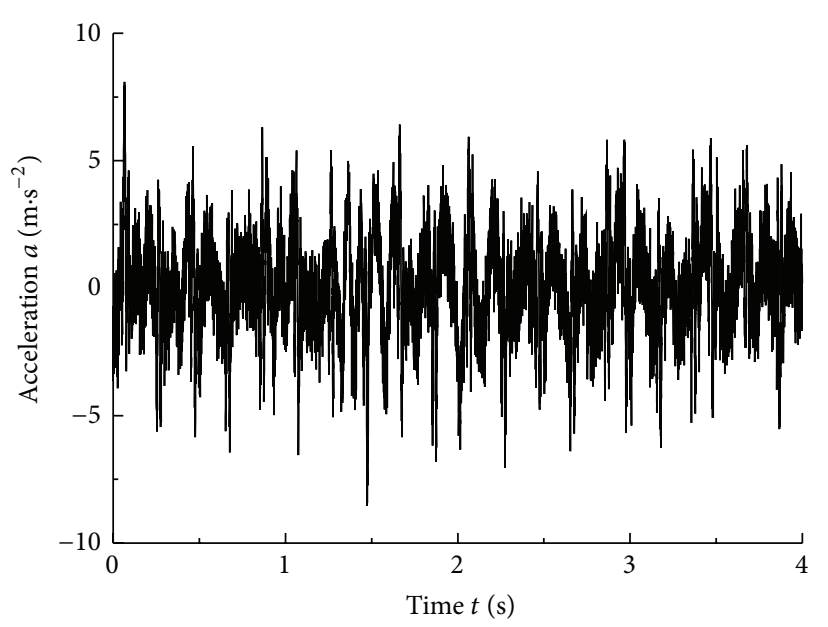

FIGURE 23: Vibration waveform of test rig experiment at the speed of $100 \mathrm{~km} / \mathrm{h}$.

\section{Conclusions}

The composition of axle box vibration signal is very complicated, which includes wheel vibration information, track status information, axle box bearing vibration information, and numerous interferences. Therefore, extracting the influential features from strong interference is a typical key issue for vehicle wheel fault detection. In the present study, an IMF superposition theory is derived, and an improved EMD analysis method is developed to investigate the problem of wheel flat fault diagnosis in railway vehicles. The effectiveness of the proposed method is evaluated by simulated vibration signals and an experimental vibration signal collected from a 


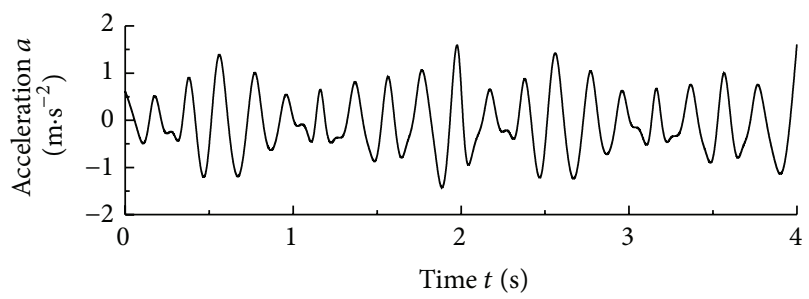

(a) The characteristic IMF selected

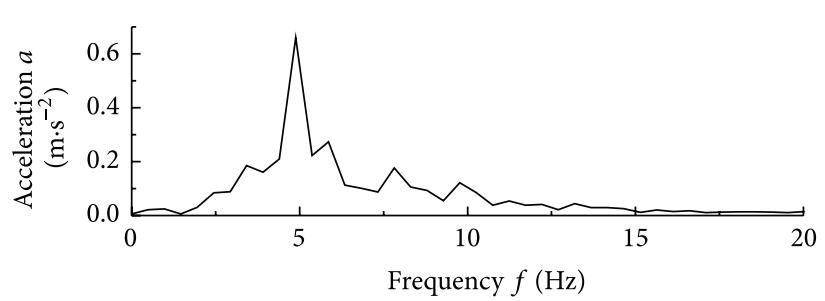

(b) Frequency spectrum

FIGURE 24: Analysis results of the signal presented in Figure 23 by improved EMD: (a) the characteristic IMF and (b) FFT spectrum.

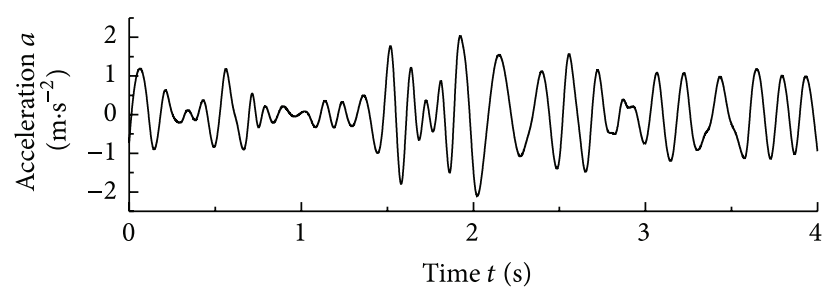

(a) The characteristic IMF selected

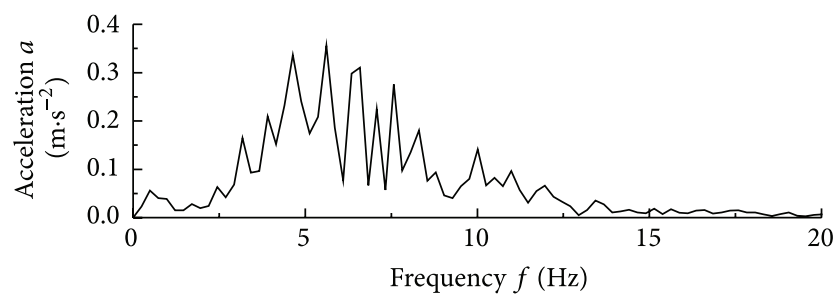

(b) Frequency spectrum

FIGURE 25: Analysis results of the signal presented in Figure 23 by EEMD: (a) the characteristic IMF and (b) FFT spectrum.

test rig. The results show that the improved EMD approach can be used to detect the fault features of interest in complex operating conditions that are invisible in EEMD.

\section{Competing Interests}

The authors declare that they have no competing interests.

\section{Acknowledgments}

This study is supported by the Fundamental Research Funds for the Central Universities, under Project no. SWJTU2682014BR001EM, and the National Natural Science Foundation of China, under Project no. 51375403.

\section{References}

[1] A. M. Remennikov and S. Kaewunruen, "A review of loading conditions for railway track structures due to train and track vertical interaction," Structural Control and Health Monitoring, vol. 15, no. 2, pp. 207-234, 2008.

[2] D. Milković, G. Simić, Ž. Jakovljević, J. Tanasković, and V. Lučanin, "Wayside system for wheel-rail contact forces measurements," Measurement, vol. 46, no. 9, pp. 3308-3318, 2013.

[3] R. Lagnebäck, Evaluation of wayside condition monitoring technologies for condition-based maintenance of railway vehicles [Ph.D. thesis], Luleå University of Technology, 2007.

[4] M. L. Filograno, P. Corredera Guillén, A. Rodríguez-Barrios et al., "Real-time monitoring of railway traffic using fiber Bragg grating sensors," IEEE Sensors Journal, vol. 12, no. 1, pp. 85-92, 2012.

[5] A. Bracciali and G. Cascini, "Detection of corrugation and wheelflats of railway wheels using energy and cepstrum analysis of rail acceleration," Proceedings of the Institution of Mechanical
Engineers, Part F: Journal of Rail and Rapid Transit, vol. 211, no. 2, pp. 109-116, 1997.

[6] V. Belotti, F. Crenna, R. C. Michelini, and G. B. Rossi, "Wheelflat diagnostic tool via wavelet transform," Mechanical Systems and Signal Processing, vol. 20, no. 8, pp. 1953-1966, 2006.

[7] A. Matsumoto, Y. Sato, H. Ohno et al., "A new measuring method of wheel-rail contact forces and related considerations," Wear, vol. 265, no. 9-10, pp. 1518-1525, 2008.

[8] M. Bocciolone, A. Caprioli, A. Cigada, and A. Collina, "A measurement system for quick rail inspection and effective track maintenance strategy," Mechanical Systems and Signal Processing, vol. 21, no. 3, pp. 1242-1254, 2007.

[9] M. Molodova, Z. Li, and R. Dollevoet, "Axle box acceleration: measurement and simulation for detection of short track defects," Wear, vol. 271, no. 1-2, pp. 349-356, 2011.

[10] Z. Li, M. Molodova, A. Nunez, and R. Dollevoet, "Improvements in axle box acceleration measurements for the detection of light squats in railway infrastructure," IEEE Transactions on Industrial Electronics, vol. 62, no. 7, pp. 4385-4397, 2015.

[11] Y. Suda, M. Hanawa, M. Okumura, and T. Iwasa, "Study on rail corrugation in sharp curves of commuter line," Wear, vol. 253, no. 1-2, pp. 193-198, 2002.

[12] B. Liang, S. D. Iwnicki, Y. Zhao, and D. Crosbee, "Railway wheelflat and rail surface defect modelling and analysis by timefrequency techniques," Vehicle System Dynamics, vol. 51, no. 9, pp. 1403-1421, 2013.

[13] Y. Chen, Z. Xing, J. Li, and Y. Qin, "The analysis of wheel-rail vibration signal based on frequency slice wavelet transform," in Proceedings of the 17th IEEE International Conference on Intelligent Transportation Systems (ITSC '14), pp. 1312-1316, Qingdao, China, October 2014.

[14] N. E. Huang, Z. Shen, S. R. Long et al., "The empirical mode decomposition and the Hilbert spectrum for nonlinear and non-stationary time series analysis," Proceedings of the Royal Society of London, vol. 454, no. 1971, pp. 903-995, 1998. 
[15] X. Zhao, T. H. Patel, and M. J. Zuo, "Multivariate EMD and full spectrum based condition monitoring for rotating machinery," Mechanical Systems and Signal Processing, vol. 27, no. 1, pp. 712728, 2012.

[16] Z. Wu and N. E. Huang, "Ensemble empirical mode decomposition: a noise-assisted data analysis method," Advances in Adaptive Data Analysis, vol. 1, no. 1, pp. 1-41, 2009.

[17] Z. Feng, M. J. Zuo, R. Hao, F. Chu, and J. Lee, "Ensemble empirical mode decomposition-based Teager energy spectrum for bearing fault diagnosis," Journal of Vibration and Acoustics, vol. 135, no. 3, Article ID 031013, pp. 1-21, 2013.

[18] Y. Lei and M. J. Zuo, "Fault diagnosis of rotating machinery using an improved HHT based on EEMD and sensitive IMFs," Measurement Science and Technology, vol. 20, no. 12, Article ID 125701, pp. 1-12, 2009.

[19] J. Zheng, J. Cheng, and Y. Yang, "Partly ensemble empirical mode decomposition: an improved noise-assisted method for eliminating mode mixing," Signal Processing, vol. 96, pp. 362374, 2014.

[20] J. Serra, "Morphological filtering: an overview," Signal Processing, vol. 38, no. 1, pp. 3-11, 1994.

[21] R. Hao and F. Chu, "Morphological undecimated wavelet decomposition for fault diagnostics of rolling element bearings," Journal of Sound and Vibration, vol. 320, no. 4-5, pp. 1164-1177, 2009.

[22] J. Zhang, R. Yan, R. X. Gao, and Z. Feng, "Performance enhancement of ensemble empirical mode decomposition," Mechanical Systems and Signal Processing, vol. 24, no. 7, pp. 2104-2123, 2010.

[23] C. Yi, J. Lin, T. Ruan, and Y. Li, "Real time cardan shaft state estimation of high-speed train based on ensemble empirical mode decomposition," Shock and Vibration, vol. 2015, Article ID 912483, 12 pages, 2015.

[24] Y. Li, M. Xu, Y. Wei, and W. Huang, "An improvement EMD method based on the optimized rational Hermite interpolation approach and its application to gear fault diagnosis," Measurement, vol. 63, pp. 330-345, 2015.

[25] Y.-F. Li, J.-X. Liu, J.-H. Lin, and Z.-J. Li, "Fault diagnosis method of railway vehicle with wheel flat based on selfadaptive multi-scale morphology analysis," Journal of Traffic and Transportation Engineering, vol. 15, no. 1, pp. 58-65, 2015.

[26] T. Mazilu, "A dynamic model for the impact between the wheel flat and rail," Mechanical Engineering, vol. 69, no. 2, pp. 45-58, 2007.

[27] T. X. Wu and D. J. Thompson, "Vibration analysis of railway track with multiple wheels on the rail," Journal of Sound and Vibration, vol. 239, no. 1, pp. 69-97, 2001. 


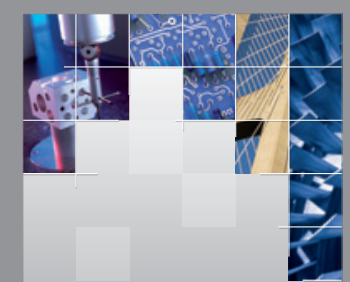

\section{Enfincering}
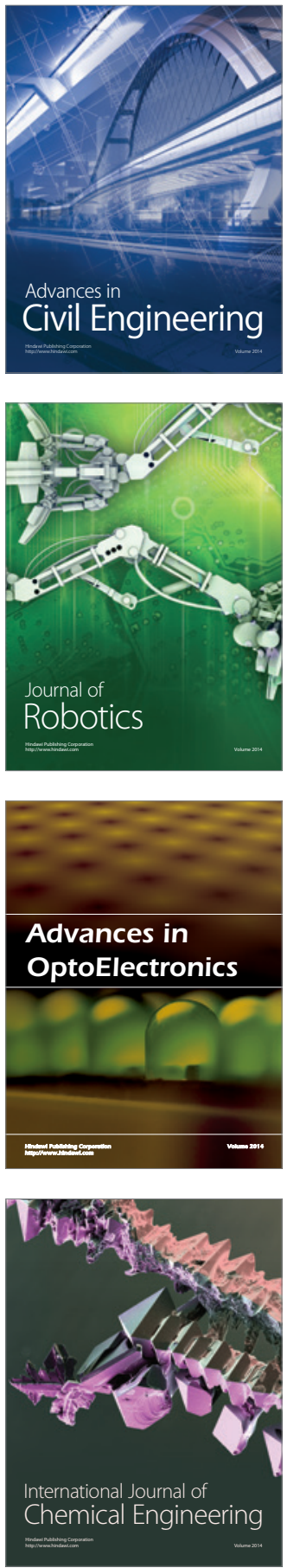

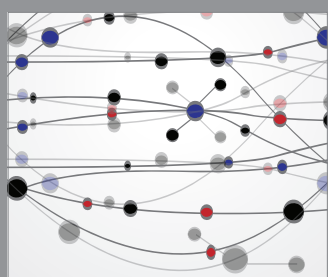

The Scientific World Journal

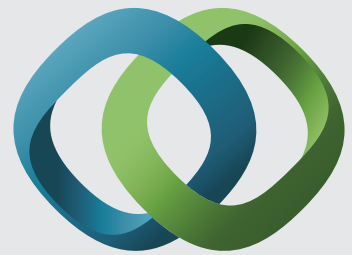

\section{Hindawi}

Submit your manuscripts at

http://www.hindawi.com
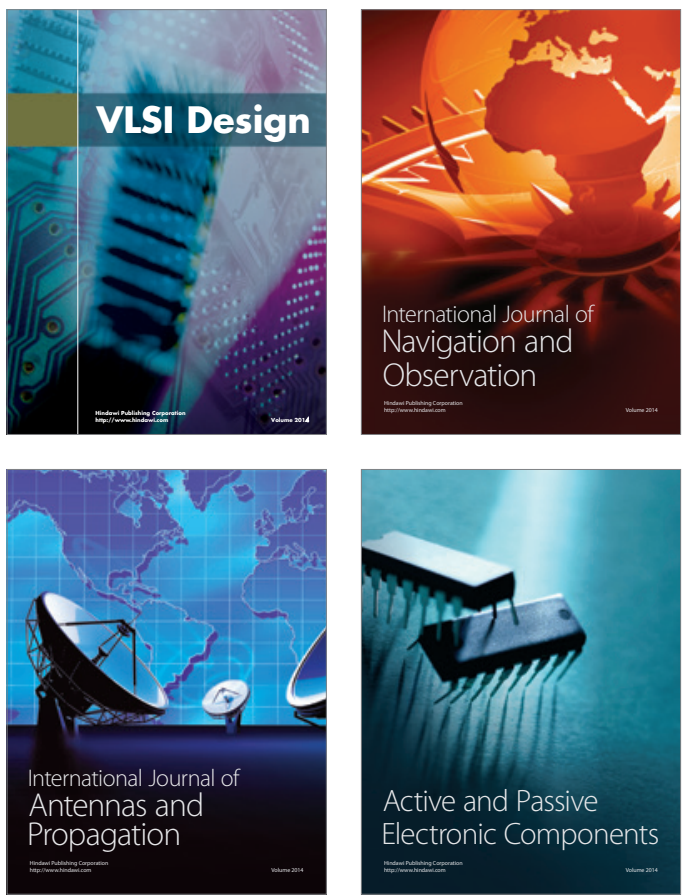
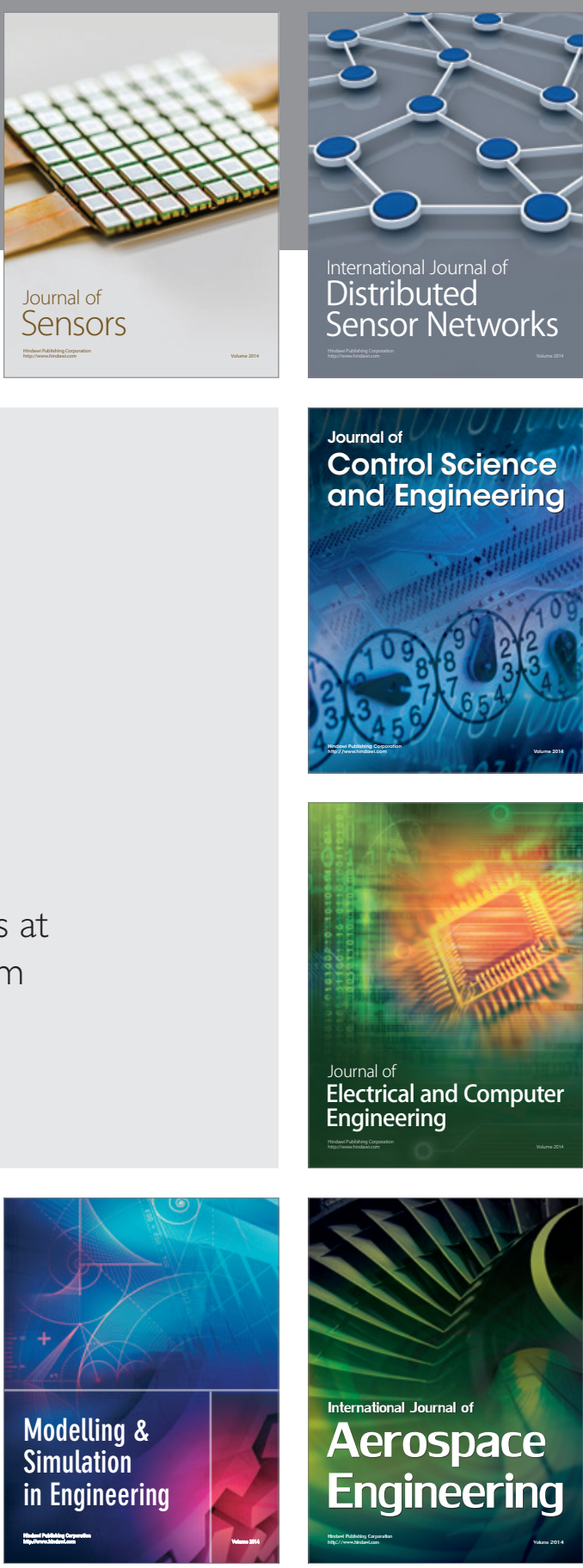

International Journal of

Distributed

Sensor Networks

Journal of

Control Science

and Engineering
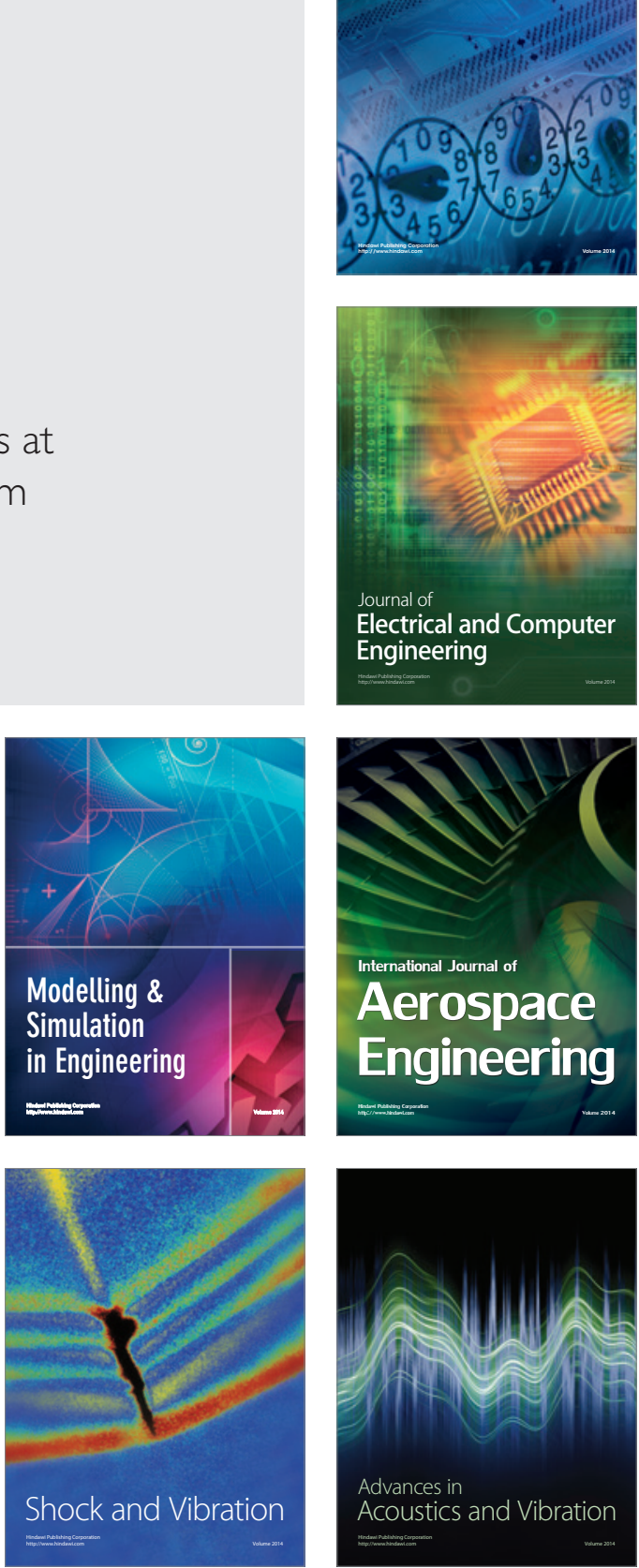